\title{
Development of an Optimised Application Protocol For Sonophoretic Transdermal Delivery of a Model Hydrophilic Drug
}

\author{
Omar Sarheed ${ }^{1}$ and Bazigha K. Abdul Rasool ${ }^{* 2}$ \\ ${ }^{I}$ Department of Pharmaceutics, RAK College of Pharmaceutical Sciences, Ras Al-Khaimah Medical and Health \\ Sciences University, Ras Al-Khaimah, UAE \\ ${ }^{2}$ Department of Pharmaceutics and Pharmacy Practice, Dubai Pharmacy College, Dubai, UAE
}

\begin{abstract}
It has now been known for over a decade that low frequency ultrasound can be used to effectively enhance transdermal drug penetration - an approach termed sonophoresis. Mechanistically, acoustic cavitation results in the creation of defects in the stratum corneum that allow accelerated absorption of topically applied molecules. The aim of this study was to develop an optimised sonophoresis protocol for studying transdermal drug delivery in vitro. To this end, caffeine was selected as a model hydrophilic drug while porcine skin was used as a model barrier. Following acoustic validation, $20 \mathrm{kHz}$ ultrasound was applied for different durations (range: $5 \mathrm{~s}$ to $10 \mathrm{~min}$ ) using three different modes $(10 \%, 33 \%$ or $100 \%$ duty cycles) and two distinct sonication procedures (either before or concurrent with drug deposition). Each ultrasonic protocol was assessed in terms of its heating and caffeine flux-enhancing effects. It was found that the best regimen was a concurrent $5 \mathrm{~min}$, pulsed $\left(10 \%\right.$ duty cycle) beam of SATA intensity $0.37 \mathrm{~W} / \mathrm{cm}^{2}$. A key insight was that in the case of pulsed beams of $10 \%$ duty cycle, sonication concurrent with drug deposition was superior to sonication prior to drug deposition and potential mechanisms for this are discussed.
\end{abstract}

Keywords: Transdermal, Sonophoresis, Caffeine, Drug delivery, Optimised, Pig skin.

\section{INTRODUCTION}

Although ultrasound has been used by physiotherapists since the 1950s [1], it only since the mid-1990s that low frequency ultrasound $(\sim 20$ to $150 \mathrm{kHz})$ has been identified as an effective means of enhancing transdermal drug delivery. Such an application of low frequency ultrasound is termed sonophoresis [2-5].

Over the last decade, considerable research has been directed towards understanding the parameters and paradigms that modulate sonophoresis. Mechanistically, it is known that acoustically-induced cavitation results in the formation of intercellular lipid channels and defects in the stratum corneum. These perturbations allow accelerated cutaneous ingress of solutes [6-8]. The extent of enhancement is determined by four principal acoustic variables - frequency, intensity, duty cycle, and duration. In general, enhancement increases with decreasing ultrasound frequency and this is due to the fact that ultrasound induces proportionally more cavitational activity at lower frequencies. Transport enhancement also increases with increasing intensity although two thresholds, both a lower cut-off intensity and upper decoupling intensity, may occur [9]. Lengthening the duration of application or using a greater duty cycle in the case of pulsed ultrasound (ie increasing the "pulse-on" time) will similarly tend to increase enhancement.

*Address correspondence to this author at the Dubai Pharmacy College, P.O. Box: 19099, Dubai, UAE; Tel: +97 150952 9878;

E-mails: bazigharasool@yahoo.com; sarheed78@gmail.com
Although the fundamental biophysical processes are now understood, it is still difficult to predict the extent of drug delivery enhancement produced by any given application of low frequency ultrasound. This is due to the fact that the extent of cavitational disordering of the stratum corneum depends upon numerous factors such as the; transducer to skin distance, transducer geometry as well as the availability and distribution of dissolved gas. Furthermore, the thermal effects of ultrasound may also act as a secondary contributor to transport enhancement [10]. This is a further confounding variable since tissue heating is difficult to predict as it is dependent upon many interactive variables, some of which exhibit positive feedback processes. Additionally, a considerable proportion of studies have used electrical resistance measurements as a surrogate marker of skin permeability [11-13] thus resulting in a relative scarcity of real permeability data within the context of sonophoresis.

The aim of the present study was to develop a suitable protocol that could be used to directly measure sonophoretic drug delivery in vitro and to determine the relative contribution of ultrasound parameters in low-frequency ultrasoundinduced percutaneous enhancement of drug transport. For this purpose, caffeine was chosen as a model hydrophilic drug while full thickness porcine skin was used as a barrier membrane. After initial acoustic source validation studies, $20 \mathrm{kHz}$ ultrasound was applied for different durations (range: $5 \mathrm{~s}$ to $10 \mathrm{~min})$ using three different modes $(10 \%, 33 \%$ and $100 \%$ duty cycles) and two distinct sonication procedures (either prior to or concurrent with drug deposition). Each ultrasonic treatment was assessed in terms of its skin surface heating and caffeine permeation-enhancing effects. The ob- 
jective was to identify the most optimal application regimen. Investigations were also conducted with respect to the effect of ultrasound on caffeine stability as well as on epidermal structure as imaged by electron microscopy.

\section{MATERIALS AND METHODS}

\section{Materials}

Caffeine, HPLC-grade acetonitrile, phosphate buffer saline tablets ( $\mathrm{pH} 7.4)$, glutaraldehyde, osmium tetraoxide $2 \%$ w/v solution, potassium dihydrogen orthophosphate and disodium hydrogen orthophosphate were purchased from Sigma-Aldrich (Poole, UK). HPLC grade glacial acetic acid was obtained from Fisher Scientific (Loughborough, UK) while absolute ethanol was purchased from Bamford Labs (Rochdale, UK). Double-distilled water was used throughout.

\section{Validation of the Ultrasound Source}

Low frequency $(20 \mathrm{kHz})$ ultrasound was generated by a VCX-500 ultrasound processor (Sonics \& Materials; Newtown CT) connected to a $3 \mathrm{~mm}$ diameter transducer probe. Since it is sometimes the case that the actual acoustic output may not match the manufacturer's specified values $[14,15]$, it was necessary to initially validate the processor's acoustic dosimetry.

Frequency measurements were undertaken using a calibrated hydrophone (B\&K 8103, Stevenage, UK) directly connected to an Infiniium oscilloscope (Agilent, Wokingham, UK) operating at $500 \mathrm{MHz}$ and $2 \mathrm{GSa} / \mathrm{s}$. However, even at the lowest possible intensity setting, the emitted ultrasound produced sufficient cavitation to interfere with the operation of the hydrophone. This problem was overcome by controlling the processor via an external DC voltage source connected through a 9-pin D-type socket. This procedure allowed the minimal acoustic energy to be reduced by $\sim 75 \%$, which facilitated effective hydrophone use. Subsequently, both the transducer tip and hydrophone were immersed in a 5-litre tank filled with distilled water. Acoustic pressure oscillations were measured at a distance of $5 \mathrm{~cm}$ from the transducer face.

The acoustic intensity emitted by the probe could, in theory, be obtained by performing a simple procedure specified by the manufacturer. Briefly, this involved adjusting the processor's amplitude dial to a selected value (range: 21 to $40 \%$ ) and subsequently reading off the power monitor readings when the probe was operating in distilled water and then in air. The difference between the two monitor readings was divided by the transducer area in order to obtain the manufacturer's intensity value. In order to validate these values, we employed a simple calorimetric method. To this end, the transducer was immersed in a Dewar-type $\left(\right.$ Dilvac $\left.^{\circledR}\right)$ thermoflask (VWR, Lutterworth, UK), filled with $500 \mathrm{ml}$ of distilled water. The ultrasound probe was immersed into the water and continuous wave ultrasound was applied for 15 minutes at different amplitude settings. Water temperatures were measured both before and after sonication. The experiment was conducted in triplicate. The following equation was used to yield the Spatial-Average Temporal-Average (SATA) intensity output:
Intensity $=\frac{\text { Mass }(m) \times \text { Specific heat capacity }(w)}{\text { Transducer area }(A)} \times(\Delta$ Temp $)$

The final validation study involved use of a laser vibrometer (Polytec GmbH, Waldbronn, Germany) to measure the mechanical vibrations of the transducer's active face [16, 17]. The transducer was isolated from the VCX500 instrument and electrically excited using a HP 33120A Arbitrary Function Generator (AFG). A $20 \mathrm{kHz}$ sinusoidal signal of peak-to-peak amplitude $10 \mathrm{~V}$ was generated using the AFG to ensure that the probe did not produce a cavitating field when recording the experimental measurements. In addition, the AFG provided a synchronisation pulse to trigger the laser vibrometry measurement system. Importantly, the surface vibration characteristic can be used to evaluate the ultrasonic pressure field using Hygen's Principle [17]. This information can be used to determine the position of any cavitating field through analysis of the pressure maxima [18].

\section{Preparation of Skin Membranes}

Porcine ears (Landrace species) were obtained from a local abattoir immediately after slaughter of the animals but before steam sterilisation of the tissue. The ears were cleaned under cold running water. The external surface of each ear was sectioned horizontally by scalpel to yield whole skin samples, of area $\sim 8 \mathrm{~cm}^{2}$. The skin sections were visually checked for integrity then stored in a frozen state $\left(-80^{\circ} \mathrm{C}\right)$ for a maximum period of 3 months [19]. This storage condition had shown no significant effect on $\mathrm{Kp}$ as a result of freezing as shown $[20,21]$. Immediately prior to the permeation studies, the porcine skin sections were thawed at room temperature and cut into smaller samples (surface area $\sim 1 \mathrm{~cm}^{2}$ ) suitable for mounting on Franz cells.

\section{Transdermal Permeation Studies}

The full-thickness skin samples were mounted in Franz diffusion cells (PermeGear, Bethlehem PA), exhibiting a diffusion-available surface area of $0.64 \mathrm{~cm}^{2}$ and a receptor compartment volume of $5.3 \mathrm{ml}$. The receptor solution consisted of PBS ( $\mathrm{pH}$ 7.4) that had been previously degassed by 5 min exposure to $35 \mathrm{kHz}$ ultrasound in a Transsonic T310 water bath sonicator (Camlab Ltd, Cambridge UK). This receiver solution was stirred at $600 \mathrm{rpm}$ and maintained at 37 $\pm 0.5^{\circ} \mathrm{C}$ by the use of a thermostatic water pump (Haake DC10, Karlsruhe, Germany) that circulated water through each chamber jacket. The skins were initially allowed to hydrate in the Franz cells for 1 hour. During this period, the Franz cells were occasionally inverted in order to allow the escape of any air bubbles that had developed on the skin underside. Subsequently, $0.5 \mathrm{ml}$ of $0.5 \% \mathrm{w} / \mathrm{v}$ caffeine solution in PBS ( $\mathrm{pH} 7.4$ ) was deposited on to each skin surface. Apart from during periods of ultrasonication or thermocouple insertion, the donor compartments were always covered with a taut layer of Parafilm ${ }^{\circledR}$ in order to minimise evaporation.

Caffeine permeation was allowed to proceed for a total period of either $24 \mathrm{~h}$ or $48 \mathrm{~h}$, depending upon the length of the lag time. At selected time intervals, a $100 \mu$ l aliquot of receiver solution was withdrawn from each receiver solution and replaced with the same volume of blank PBS solution. Permeant amounts in the withdrawn solutions were determined by HPLC. The caffeine concentration values derived 
for each aliquot were corrected for the progressive dilutions occurring during the course of the experiment, as described previously $[22,23]$. Each individual experiment consisted of between 6 to 8 replicate runs.

\section{Ultrasonication Protocols}

Two types of ultrasound exposure protocols were employed - pretreatment and concurrent applications.

For pretreatment experiments, sonication was undertaken immediately after hydration of the skin but prior to the topical application of the caffeine-containing solution. This step involved the deposition of $0.5 \mathrm{ml}$ of PBS ( $\mathrm{pH} \mathrm{7.4)} \mathrm{in} \mathrm{each}$ donor compartment. The transducer, aligned perpendicular to the skin surface, was immersed in this solution with its active tip face located $5 \mathrm{~mm}$ above the skin surface. The skin was then exposed to different ultrasound treatment regimens with the spatial- average temporal-peak (SATP) intensity fixed at $3.7 \mathrm{~W} / \mathrm{cm}^{2}$. Application of continuous wave ultrasound was conducted for a total duration of either 0s (control), 5s, 30s, 120s, or 600s. Application of pulsed ultrasound in 1 s on: 2 s off mode ( $33 \%$ duty cycle) was conducted for a total duration of 30 s or 120 s. The application of ultrasound in $1 \mathrm{~s}$ on: $9 \mathrm{~s}$ off mode (10\% duty cycle) was also performed over 300s or 1200 s.

For concurrent ultrasound studies, sonication was undertaken immediately after topical deposition of the caffeinecontaining solution. The transducer was clamped into position in the same manner as described above and activated using the same duration-mode combinations listed above.

Skin surface temperature measurements were undertaken during the course of each ultrasound application. These measurements were conducted with a HI 8757 microprocessor-controlled thermometer (Hanna instruments Ltd., Leighton Buzzard, UK).

\section{Contribution of Thermal Effects to Sonophoretic- Enhanced Transport}

To differentiate between the thermal and the non-thermal effects on the drug transport, heat treatment was used to compare with the ultrasound treated group. Permeation experiments were performed by replacing the ultrasound transducer by an electrical resistance connected to an AC generator. The electrical power delivered by the generator was controlled to reproduce the temperature versus time profile of the donor solution observed during sonication for $5 \mathrm{~min}$. The temperature closed to the skin would be heated and raised most greatly to $42.6^{\circ} \mathrm{C}$. Generally-speaking it is believed that $45^{\circ} \mathrm{C}$ is a cut-off temperature beyond which irreversible changes occur in the skin [24].

\section{Caffeine Stability Studies}

The aim of these experiments was to determine whether ultrasonic exposure degrades caffeine. For this purpose, $0.5 \mathrm{ml}$ aliquots of $0.5 \% \mathrm{w} / \mathrm{v}$ caffeine solution in PBS ( $\mathrm{pH} 7.4$ ) were deposited on porcine skin samples mounted on Franz cells. Each donor solution was sonicated for 600s with continuous wave energy at an intensity of $3.7 \mathrm{~W} / \mathrm{cm}^{2}$. Each topical solution was assayed for caffeine by HPLC both before and after sonication. The experiment was performed in triplicate.

\section{HPLC Assay}

Analysis of caffeine samples was performed on a HPLC system (Finnigan SpectraSystem ${ }^{\circledR}$, ThermoElectron Corp.) equipped with a solvent degasser (Model SCM1000), an autosampler (Model AS3000) and UV photodiode array detector (Model 6000). Samples were eluted on a $\mathrm{C}_{18^{-}}$ Phenomenex ${ }^{\circledR}$ column $(150 \mathrm{~mm} \times 4.6 \mathrm{~mm} ; 5 \mu \mathrm{m})$ using a mobile phase consisting of consisting of $0.05 \mathrm{M}$ acetic acid: acetonitrile $(90: 10)$. The elution parameters were a flow rate of $1 \mathrm{ml} / \mathrm{min}$ and an injection volume of $20 \mu \mathrm{l}$. The detection wavelength was $272 \mathrm{~nm}$. Calibration of the system was achieved through the use of external standards. The analytical parameters for this assay were as follows: retention time was 5.5 minutes, limit of detection was $0.5 \mu \mathrm{g} / \mathrm{ml}$, and reproducibility relative standard deviation was $2 \%$.

\section{Scanning Electron Microscopy}

Electron microscopy was used to visualise the effect of a concurrent 300 s pulsed ultrasound (10\% duty cycle) application on epidermal structure. In order to achieve this, whole porcine skin samples were mounted in Franz cells, treated with caffeine solution and subjected to this sonication regimen in the manner described above (sections 2.4 and 2.5). Other skin samples were subjected to a control treatment that involved topical caffeine solution deposition but no ultrasound application. At 1 hour after caffeine application, the skin samples were removed from the diffusion cells and a scalpel was used to excise tissue strips of dimensions $\sim 1 \mathrm{~mm}$ $\mathrm{x} 2 \mathrm{~mm}$ from the central area of each sample.

The first stage of fixation involved immersing the skin strips for 1 hour at room temperature in a solution of $2.5 \%$ glutaraldehyde in $0.1 \mathrm{M}$ phosphate buffer ( $\mathrm{pH}$ 7.4). Subsequently, the samples were transferred to a temperaturecontrolled room maintained at $4^{\circ} \mathrm{C}$ where they were immersed overnight in $0.1 \mathrm{M}$ of phosphate buffer. Each skin section was then washed three times with $0.1 \mathrm{M}$ of phosphate buffer. A $1 \%$ buffered solution of osmium tetraoxide was prepared by mixing, at a 1:1 ratio, an aqueous solution of $2 \%$ w/v osmium tetraoxide with $0.05 \mathrm{M}$ phosphate buffer. The skin samples were immersed in this buffer for 1 hour at room temperature. Each skin sample was then serially washed six times with distilled water in order to remove excess residues of osmium tetraoxide.

Progressive dehydration of the skin was undertaken at room temperature. To this end, the skin strips were initially washed for one minute in $30 \%$ aqueous ethanol and then washed for a further minute in $50 \%$ aqueous ethanol. Subsequently, the tissues were immersed in $70 \%$ aqueous alcohol for one hour, $90 \%$ aqueous alcohol for 10 minutes and finally absolute alcohol for 10 minutes. The samples were dried in a model CPD750 critical point dryer (Emscope, Ashford, UK). The dried specimens were gold-coated on a K575X Peltier-cold stage sputter-coater (Emitech, Ashford, UK). Specimens were then observed under a Philips 500 scanning electron microscope (Eindhoven, Netherlands) using a $3 \mathrm{kV}$ or $6 \mathrm{kV}$ accelerating voltage. Photographs were taken of suitably representative skin regions. 


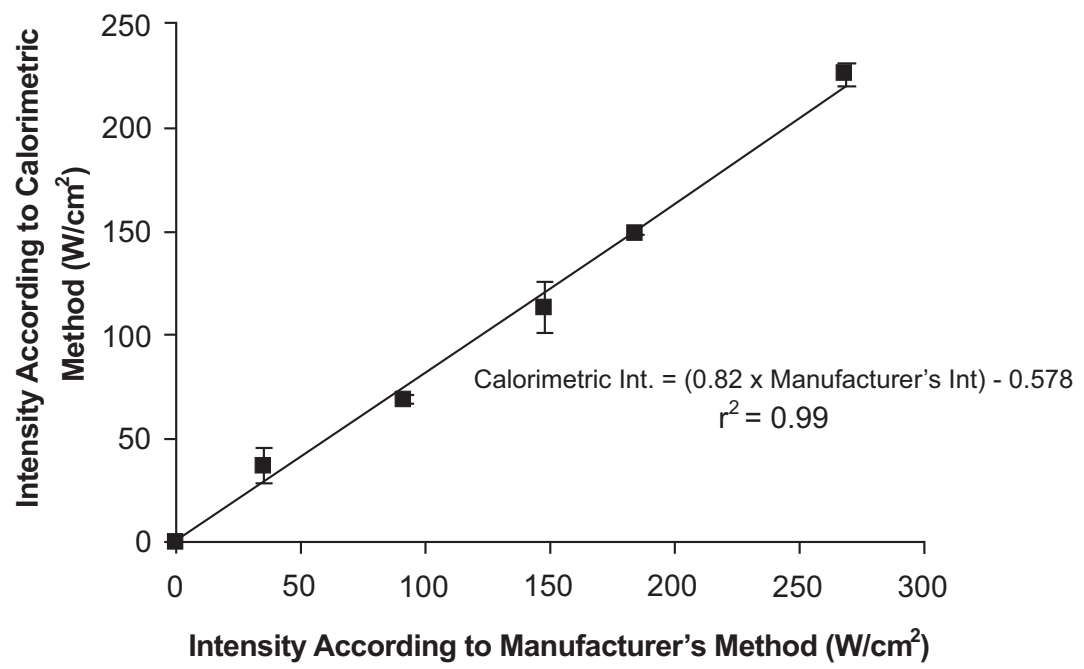

Fig. (1). Acoustic intensity calibration plot for the VCX-500 ultrasonic processor. Error bars represent standard deviation values; $n=3$.

(a)

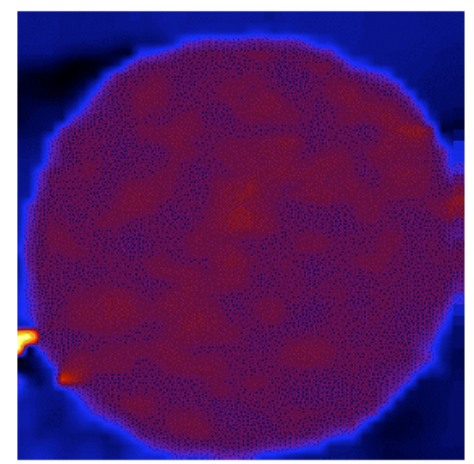

(b)

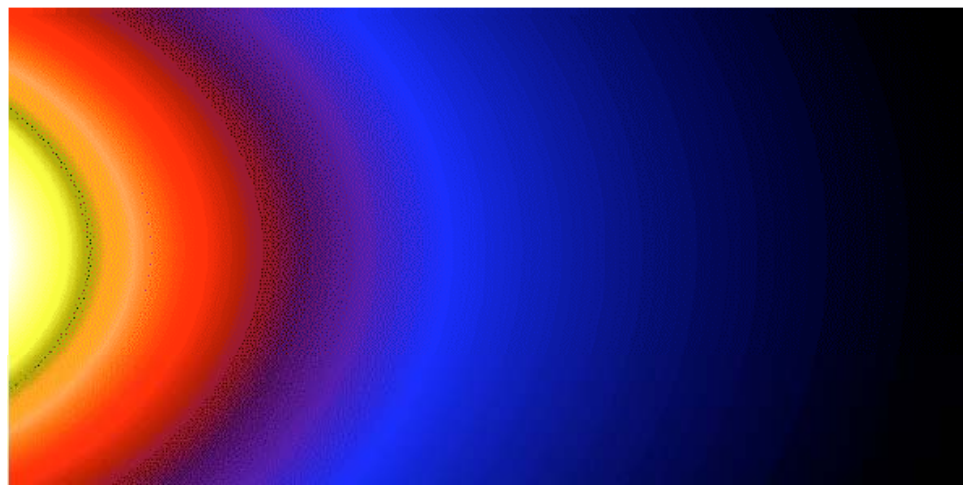

Fig. (2). The surface displacement characteristics of the active transducer tip in terms of: (a) Magnitude, (b) Details of predicted acoustic pressure field close to probe tip. The $\mathrm{x}$ and $\mathrm{y}$ axes represent spatial dimensions (arbitrary values).

\section{RESULTS}

\section{Validation of the Ultrasound Source}

Hydrophone measurements confirmed that the acoustic frequency was constant at $19.88 \mathrm{kHz}$, which is just $0.6 \%$ below the manufacturer's indicated value. With respect to intensity, Fig. (1) shows that there was a good linear correlation $\left(\mathrm{r}^{2}=0.993\right)$ between the measured intensity values and the manufacturer's intensity values. Yet from the equation shown in Fig. (1), the calorimetrically-measured intensities were somewhat lower $(\sim 18 \%)$ than that specified by the manufacturer hence providing evidence for the necessity of validation in this type of study.

Fig. (2a) illustrates the experimentally measured surface displacement characteristic across the probe surface, as imaged using laser vibrometry. It can be seen that despite some spatial variability, the active transducer face exhibited a relatively homogenous surface displacement magnitude. Moreover, the corresponding phase characteristic demonstrated that the transducer behaviour was close to the desired piston-like operation (data not shown). By extrapolating this data [17] the pressure field distribution generated by the probe was 

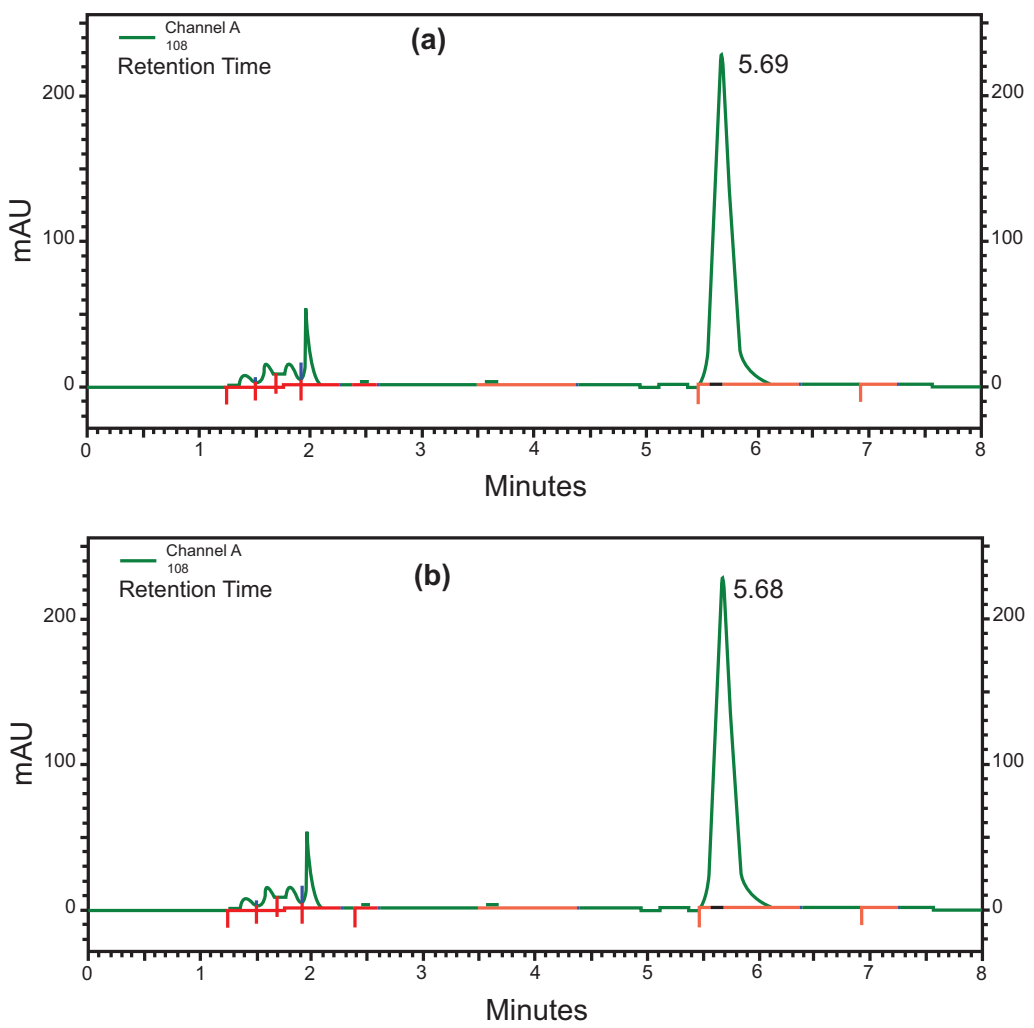

Fig. (3). Caffeine stability chromatograms shows the effect of continuous wave ultrasound for $600 \mathrm{~s}$ at $3.7 \mathrm{~W} / \mathrm{cm}^{2}$ (a) before ultrasound exposure and (b) after ultrasound exposure $(n=3)$.

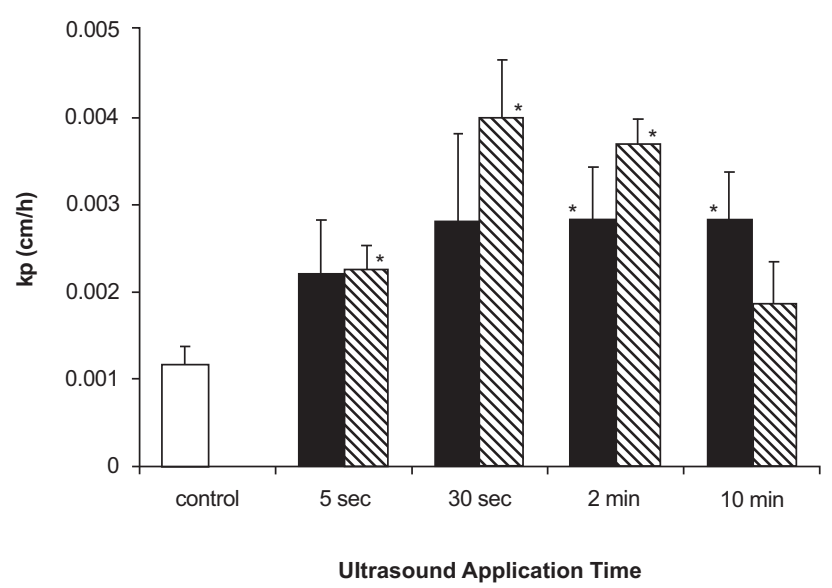

Fig. (4). The influence of continuous wave ultrasound on the transdermal permeability of caffeine. Black columns indicate concurrent ultrasound exposure while diagonal pattern columns indicate ultrasound pre-treatment. Error bars represent S.E.M. values, $n \geq 6$. * indicates significantly different from control as determined by t-test $(\mathrm{P} \leq 0.05)$.

evaluated. Fig. (2b) illustrates that the high intensity region is in close proximity to the probe tip.

\section{Ultrasound Effect on Caffeine Stability}

It was found that exposing topical caffeine solution to continuous wave ultrasound at $3.7 \mathrm{~W} / \mathrm{cm}^{2}$ for $600 \mathrm{~s}$ did not cause any drug degradation (Fig. 3a and b). The HPLC method showed a recovery percentage of $100 \%$. Since this represented the maximal energy used in our experiments, we could exclude the possibility of caffeine degradation occurring in the present study.

\section{Transport Data}

\section{Continuous Wave Ultrasound}

Fig. (4) shows the influence of continuous wave ultrasound application on the steady state permeation of caffeine through full thickness skin. It can be seen that in the absence of ultrasound, the mean $\mathrm{k}_{\mathrm{p}}$ was $11.7 \times 10^{-4} \mathrm{~cm} / \mathrm{h}$. This is very close to the mean value of $13.6 \times 10^{-4} \mathrm{~cm} / \mathrm{h}$ that we have measured previously in our lab for passive caffeine transport [22]. These figures are somewhat higher than the $k_{p}$ value of $2.5 \times 10^{-4} \mathrm{~cm} / \mathrm{h}$ recorded by [25] for caffeine transport across full-thickness pig ear skin. Differences in vehicle composi- 


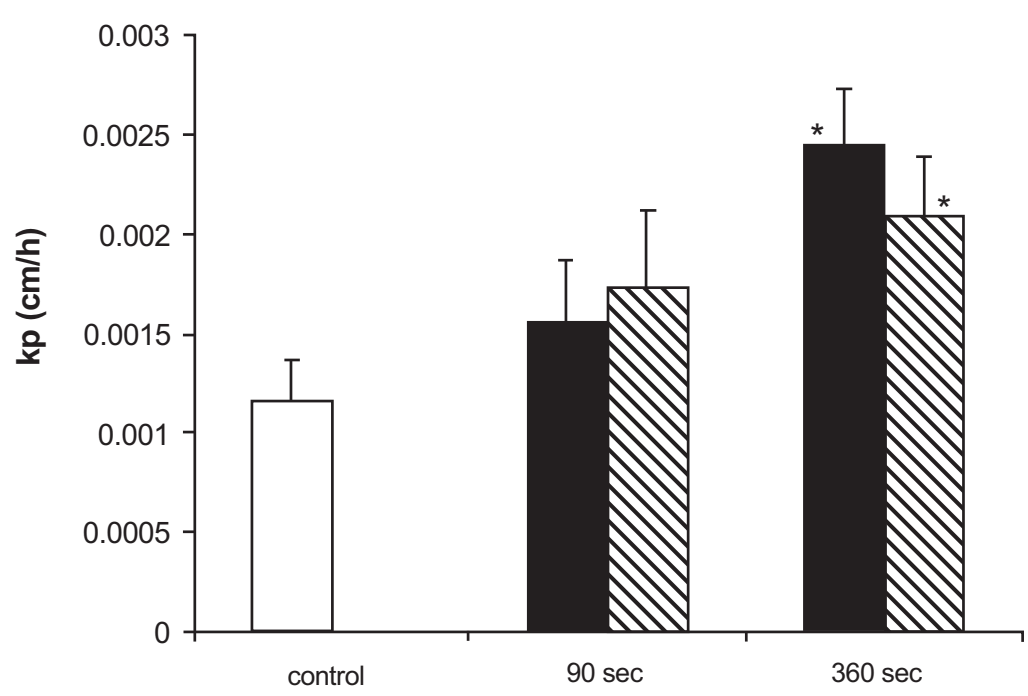

Ultrasound Application Time

Fig. (5). The influence of pulsed ultrasound (33\% duty cycle) on transdermal caffeine permeability. Black columns indicate concurrent ultrasound exposure while diagonal pattern columns indicate ultrasound pre-treatment. Error bars represent S.E.M. values, $n \geq 6$. * indicates significantly different from control as determined by $\mathrm{t}$-test $(\mathrm{P} \leq 0.05)$.

tion, experimental conditions as well as porcine species may explain this discrepancy.

In the current study, concurrent application of ultrasound for periods of 5, 30, 120 or 600 s resulted in an approximate doubling of transdermal caffeine permeation, although the effect was only statistically significant for the two lengthiest applications. Sonication for 0, 5, 30, 120 and 600s resulted in mean skin surface temperatures of $31.0^{\circ} \mathrm{C}, 31.7^{\circ} \mathrm{C}, 37.5^{\circ} \mathrm{C}$, $39.6^{\circ} \mathrm{C}$ and $43.0^{\circ} \mathrm{C}$ being attained immediately after sonication at these respective time points. It should be noted that attenuation can result in much greater heating occurring in the underlying skin layers. Hence, these surface temperatures may underestimate the total extent of tissue heating.

In the ultrasound pre-treatment studies, sonication for 5 , 30, 120 and 600s enhanced steady state caffeine permeation in comparison to passive permeation by $1.9,3.4,3.1$ and 1.5fold respectively (Fig. 4). These enhancement effects were statistically significant except for the 600s application, which was in significant $(\mathrm{P} \leq 0.05)$. Sonication for $0,5,30,120$ and $600 \mathrm{~s}$ resulted in respective mean skin surface temperatures of $31.0^{\circ} \mathrm{C}, 31.8^{\circ} \mathrm{C}, 37.5^{\circ} \mathrm{C}, 45.2^{\circ} \mathrm{C}$ and $57.2^{\circ} \mathrm{C}$ being attained immediately after irradiation. Hence, ultrasound pretreatment produced greater heating of skin than the same duration of concurrently applied ultrasound. This may be due to the absence of dissolved caffeine in the solution and / or that these solutions were degassed before being used in the transport studies. Either of these factors can influence the rate of acoustic attenuation in the solution-filled donor compartment.

\section{Pulsed Ultrasound with a 33\% Duty Cycle}

Fig. (5) shows the influence of pulsed ultrasound applications (33\% duty cycle) on steady state cutaneous transport of caffeine. The graphs show that concurrent sonication for $90 \mathrm{~s}$ and $360 \mathrm{~s}$ increased the mean $\mathrm{k}_{\mathrm{p}}$ by 1.3 times and 2.09 times respectively. Of these, only the lengthier application produced significantly increased permeation enhancement. Im- mediately after $90 \mathrm{~s}$ and 360 s of ultrasonication, skin surface temperatures were $35.3 \pm 0.8{ }^{\circ} \mathrm{C}$ and $39.5 \pm 2.2^{\circ} \mathrm{C}$ respectively.

For the ultrasound pre-treatment approach, 90s and 360s of sonication enhanced caffeine permeation by 1.4 -fold and 1.78-fold respectively. Again, only the $\mathrm{k}_{\mathrm{p}}$ elevation induced by the greater duration was statistically significant. Skin surface temperatures immediately following sonication were $34.4 \pm 0.9^{\circ} \mathrm{C}$ and $37.2 \pm 1.9^{\circ} \mathrm{C}$ respectively.

\section{Pulsed Ultrasound with a 10\% Duty Cycle}

Fig. (6) depicts the effects of pulsed ultrasound $(10 \%$ duty cycle) on transcutaneous caffeine delivery. Concurrent application of ultrasound for 300s yielded a significant 3.88fold increase in mean caffeine permeability, relative to control conditions. This $300 \mathrm{~s}$ application resulted in a postsonication skin surface temperature of $33.9 \pm 0.7^{\circ} \mathrm{C}$. A lengthier sonication period of 1200 s yielded a significant 3.03-fold enhancement in drug permeability and was associated with a final temperature of $34.1 \pm 0.7^{\circ} \mathrm{C}$ at the skin surface.

Use of the pre-treatment method for 300s produced an insignificant 2.27-fold enhancement in mean caffeine permeability, relative to passive conditions. This was associated with a skin surface temperature of $33.7 \pm 0.6{ }^{\circ} \mathrm{C}$ when the ultrasound was switched off. Increasing the ultrasound duration to 1200 s induced a significant 1.92-fold elevation in caffeine permeation. The post-sonication skin temperature was $34.2 \pm 0.5^{\circ} \mathrm{C}$.

\section{Selection of an Optimal Sonication Protocol}

Table 1 lists all the tested sonication regimens that produced significant increases in caffeine permeation while not elevating skin surface temperatures beyond $5^{\circ} \mathrm{C}$ over the control levels $\left(31^{\circ} \mathrm{C}\right)$. It can be seen that the $10 \%$ pulsed beam applied concurrently with caffeine treatment over 300 s yielded a 3.88-fold enhancement in drug transport coupled 


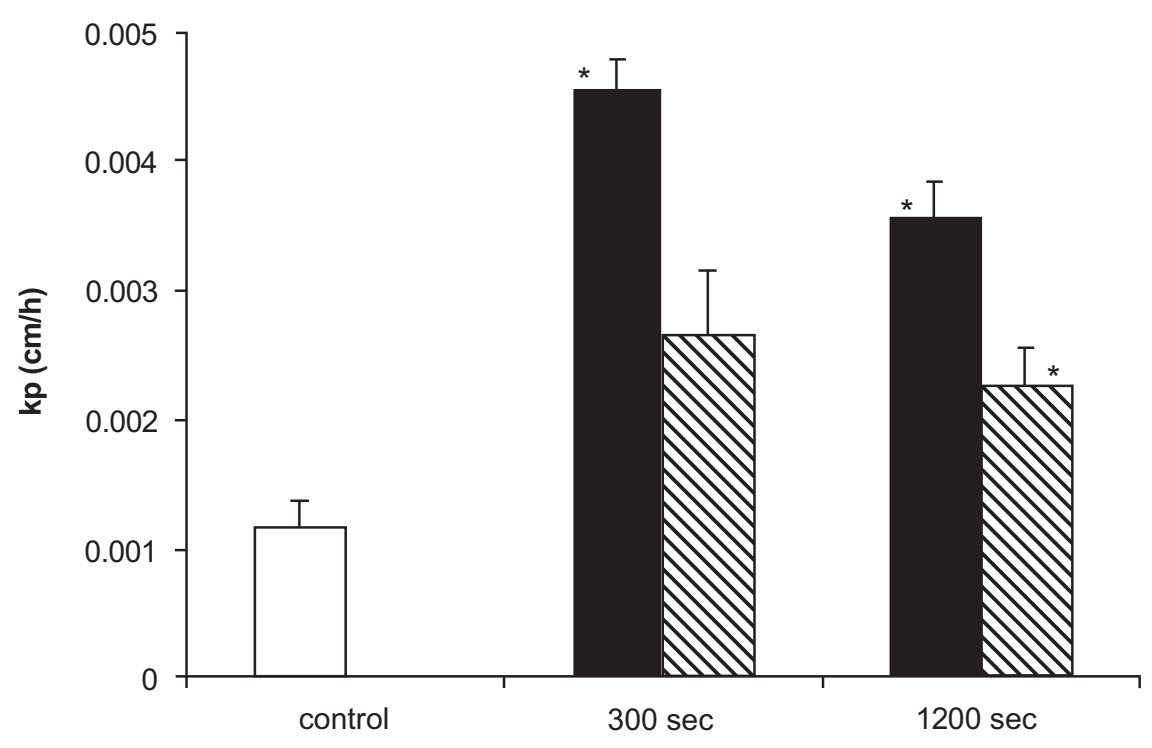

\section{Ultrasound Application Time}

Fig. (6). The influence of pulsed ultrasound (10\% duty cycle) on the transdermal permeability of caffeine. Black columns indicate concurrent ultrasound while diagonal pattern columns indicate ultrasound pre-treatment. Error bars represent s.e.m. values, $n \geq 6$. $*$ indicates significantly different from control as determined by t-test $(\mathrm{P} \leq 0.05)$.

Table 1. Permeabilising Sonication Regimens that Produced Significant Increases in Caffeine Permeation

\begin{tabular}{|c|c|c|c|c|}
\hline Application & $\begin{array}{c}\text { Total Duration } \\
(\mathbf{s})\end{array}$ & $\begin{array}{c}\text { Duty Cycle } \\
(\boldsymbol{\%})\end{array}$ & Mean Temperature Rise $\left({ }^{\circ} \mathbf{C}\right)$ & Enhancement Ratio \\
\hline \hline Pretreatment & 5 & 100 & 0.8 & 1.90 \\
\hline Pretreatment & 90 & 33 & 2.9 & 3.7 \\
\hline Concurrent & 300 & 10 & 3.1 & 3.88 \\
\hline Concurrent & 1200 & 10 & 3.2 & 1.92 \\
\hline Pretreatment & 1200 & 10 & & \\
\hline
\end{tabular}

with a temperature elevation of only $2.9^{\circ} \mathrm{C}$. The combination of a relatively brief application period, high enhancement ratio and moderate heating makes this regimen probably optimal for performing sonophoretic delivery studies. The Spatial-average temporal-average (SATA) intensity of this beam, given by multiplying the SATP intensity value by the duty cycle, was $0.37 \mathrm{~W} / \mathrm{cm}^{2}$. Fig. (7) presents the full cumulative penetration time plot for this selected sonication protocol. Interestingly, ultrasonication did not greatly affect the lag time.

\section{Contribution of Thermal Effects to Sonophoretic- Enhanced Transport}

To separate out the thermal contribution of US application to the enhancement of transdermal delivery it was necessary to investigate the thermal effect of sonophoresis via temperature control as prescribed in the methods section. The caffeine transport results are summarized in Fig. (8). Clearly, sonophoresis at $20 \mathrm{kHz}$ (concurrent; 300s; $10 \%$ duty cycle), and at an intensity of $0.37 \mathrm{~W} / \mathrm{cm}^{2}$ as well as heat alone significantly enhances skin permeability by 3.88 times and 1.60 times respectively. However, the temperature 'con- trol' experiment revealed that $\sim 40 \%$ of this improvement could be attributed solely to a thermal effect. The figure is somewhat higher than the thermal contribution value of $\sim 25 \%$ reported by Merino G et al. [10]. Differences in drug and vehicle composition, experimental conditions as well as porcine species may explain this discrepancy.

\section{Morphological Effects}

Fig. (9) shows an electron micrograph-derived surface view of human skin, 1 hour after deposition of the topical caffeine solution. It can be seen that without ultrasonication, the corneocytes on the skin surface remained relatively flat, intact and confluent. However, application of the selected sonication regimen (concurrent; 300s; 10\% duty cycle) caused the corneocytes to become partially detached from each other and develop defects and tears. Additionally, it appears that the entire layer of corneocytes has been lifted up and undergone considerable disordering (Fig. 10).

\section{DISCUSSION}

Screening of the different sonication regimens showed that each application produced differential heating and caffeine flux enhancement effects. As previously mentioned, 


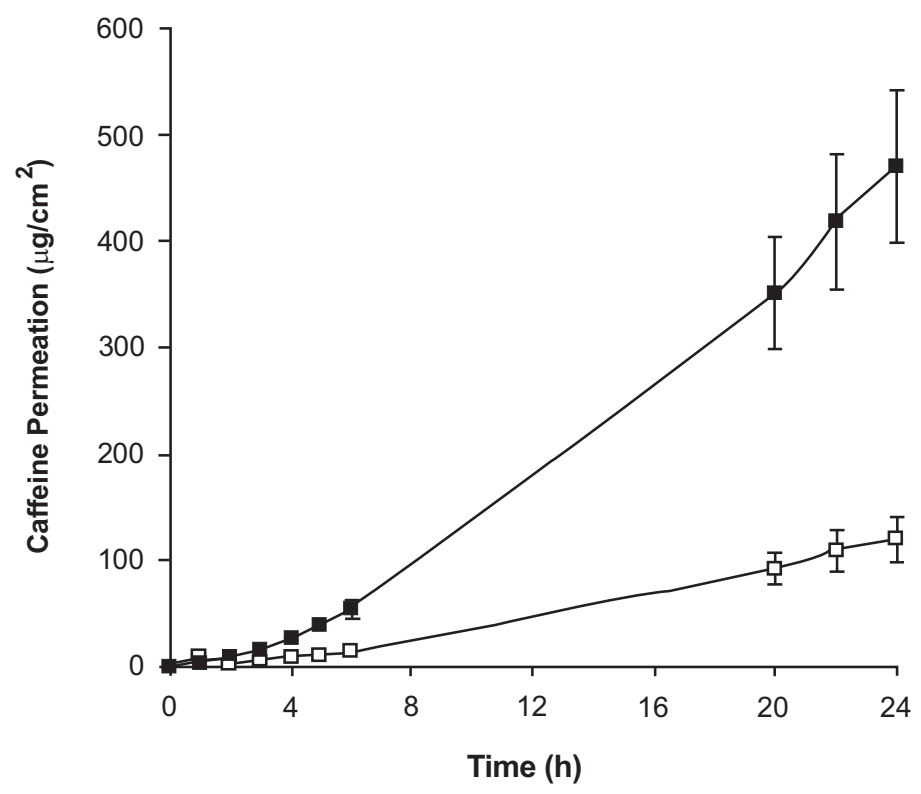

Fig. (7). The effect of 300s of concurrently-applied pulsed ultrasound (10\% duty cycle) on the cumulative permeation of caffeine across porcine skin. Empty and filled squares denote passive and sonophoretic conditions, respectively. Error bars represent S.E.M. values, $n \geq 6$.

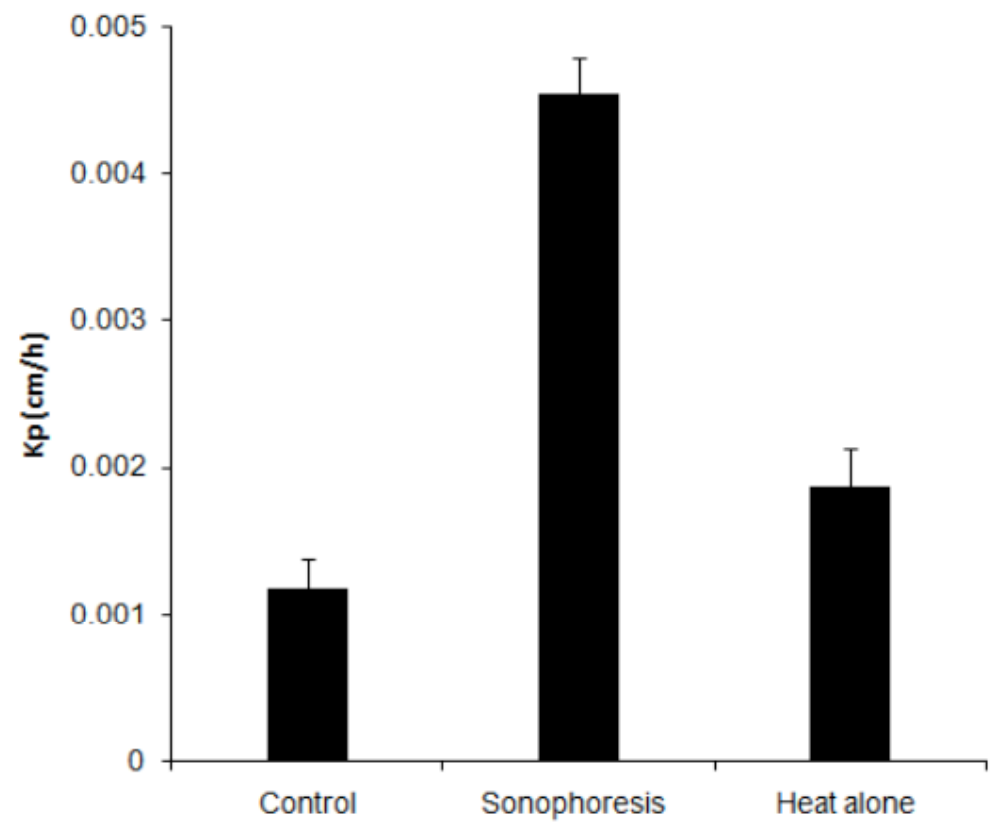

Fig. (8). The effect of 300 s of concurrently-applied pulsed ultrasound (10\% duty cycle) and heat alone on the transdermal caffeine permeability across porcine skin. Error bars represent S.E.M. values, $n \geq 6$.

the magnitude of these phenomena would have been notoriously difficult to predict from theory due to the complex and interactive nature of the multiple variables involved.

Out of all the tested sonication protocols, we found that the best was a 300 s, concurrently-applied, pulsed (10\% duty cycle) output with a SATA intensity of $0.37 \mathrm{~W} / \mathrm{cm}^{2}$. This regimen was optimal for performing sonophoretic drug delivery studies as it was relatively brief, induced minimal skin heating yet produced an almost 4-fold increase in steady state caffeine flux. Barrier permeabilisation was due to perturbation of the stratum corneum, probably mostly induced by acoustic cavitation. However, in vivo, the stratum corneum is likely to recover as the underlying viable epidermal cells would undergo upward movement and desquamation.
A highly positive aspect of our selected sonication protocol was its brief duration. In an important study, Boucaud and co-workers $[26,27]$ also used a $20 \mathrm{kHz}$ output with a $10 \%$ duty cycle and found that this beam facilitated a 3.73fold increase in caffeine permeation across dermatomed human skin. However, this was achieved by using a SATA intensity of $2.5 \mathrm{~W} / \mathrm{cm}^{2}$ and sonicating for a full hour. Others [28] experimented with continuous wave $40 \mathrm{kHz}$ ultrasound exhibiting a SATA intensity of $0.44 \mathrm{~W} / \mathrm{cm}^{2}$ (SATA) and reported a 3.81-fold increase in in vitro caffeine flux across hairless mouse skin. Although ultrasonication did not elevate skin temperature, the application lasted for 5 hours. Clearly, it is desirable to develop an in vitro transdermal delivery system that involves ultrasound application lasting several 
(a)

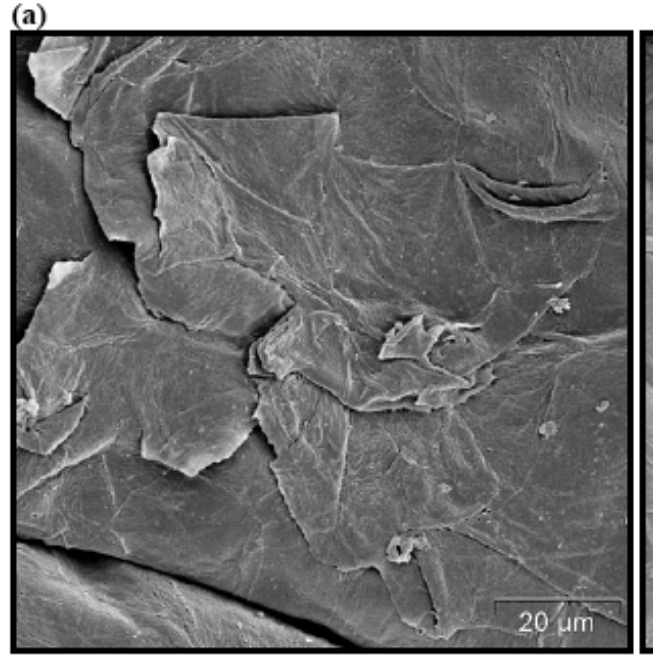

(b)

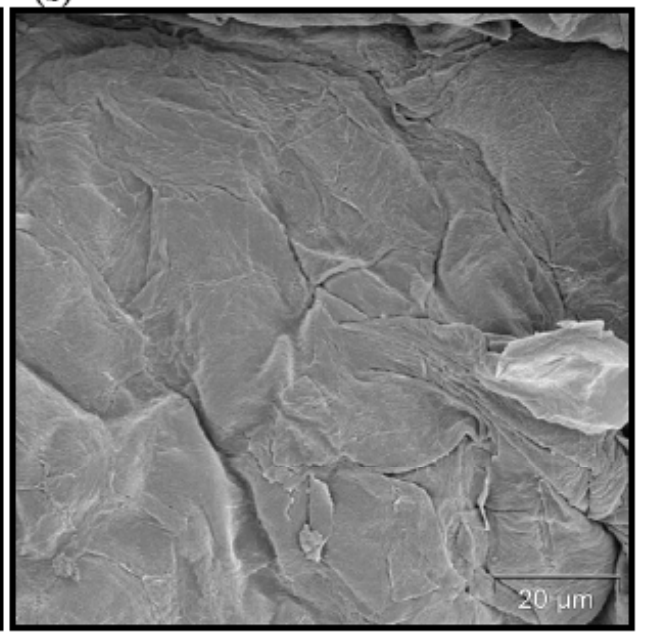

Fig. (9). Representative surface view of porcine skin (original magnification x1000) (a) at 1 hour after deposition of topical caffeine solution and (b) at 24 hour after deposition of topical caffeine solution.

(a)

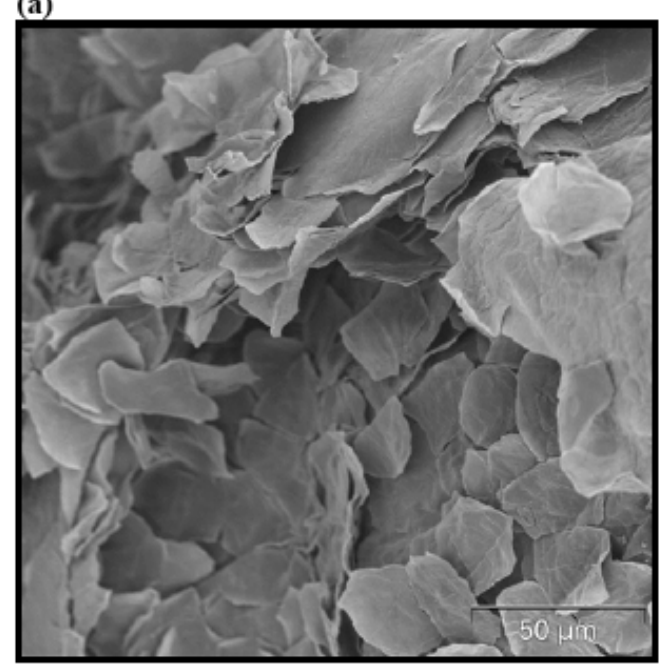

(b)

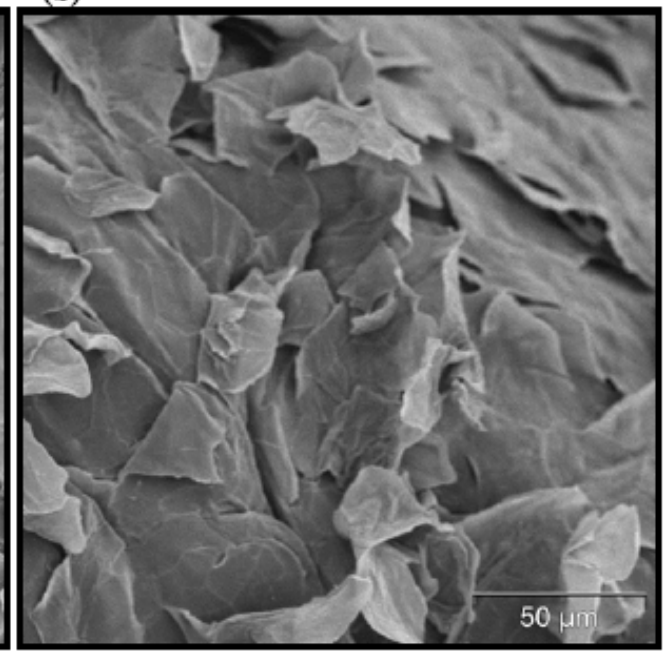

Fig. (10). Representative surface view of porcine skin (original magnification x 500) after deposition of topical caffeine solution with concurrent exposure to pulsed ultrasound (10\% duty cycle) for 300s. (a) At 1 hour after treatment (b) at 24 hour after treatment.

minutes rather than hours as this will have greater clinical relevance.

Interestingly, in the case of exposure to beams with a $10 \%$ duty cycle, both concurrent applications were more permeabilising than the pre-treatment applications of the same duration (See Fig 6). The differences were significant for sonication for 300 s (t-test: $P=0.006$ ) and for sonication for 1200 s (t-test: $P=0.005)$. There are two potential explanations for this. One possibility is that the cavitational-induced microstreaming and mixing is necessary in order to allow for maximal caffeine flux through ultrasonically-compromised stratum corneum. In other words, acoustic cavitation not only produces defects in the stratum corneum but also creates secondary microcurrents that further accelerate drug flux through these defects. An alternative mechanism relates to the fact that the stratum corneum exhibits some elasticity [29]. It is possible that after the ultrasound is switched off, at least some of the cavitationally-induced pores partially close over (Prof Richard Guy: personal communication). Either or both of these explanations would explain why the concurrent application was superior to pre-application for $10 \%$ pulsed beams. It is also possible that the time between sonication and drug application in the pretreatment application may influence on the efficiency of drug transport through the skin. However, this is an area needs to be further investigated.

It is noteworthy that in the case of the $33 \%$ and $100 \%$ duty cycle beams, there was no consistent difference in sonophoretic efficacy between the pre-treatment and concurrent regimens. This is likely due to the fact that these beams produced large and variable heating effects. Such thermal components of skin permeabilisation would tend to mask those due to cavitation. In contrast, for all four $10 \%$ duty cycle beams, skin surface temperature elevations were consistently small (between $2.7^{\circ} \mathrm{C}$ to $3.2^{\circ} \mathrm{C}$ ) as the skin cooled during the relatively lengthy "ultrasound off" times.

The skin permeability enhancement induced by sonophoresis can be attributed to both thermal and nonthermal effects [30]. To clarify the root of permeability a control tempera- 
ture experiment was conducted which revealed $\sim 40 \%$ of skin enhancement could be caused due to the increase in temperature.

Additionally the skin permeability increased significantly $(\mathrm{p} \leq 0.05)$ almost 2 times when heated to $42.6{ }^{\circ} \mathrm{C}\left(10{ }^{\circ} \mathrm{C}\right.$ increase). The literature supports the observation and suggests that for every $10{ }^{\circ} \mathrm{C}$ increase in temperature leads to doubling of skin permeability [31].

However, it is evident that the enhancement effect of sonophoresis goes beyond that provoked by a simple temperature increase; alternatively cavitation is proposed to be main mechanism for skin enhancement [26, 27].

\section{CONCLUSIONS}

Our study showed that the most practical regimen for sonophoretic drug delivery was a concurrent $5 \mathrm{~min}$, pulsed ( $10 \%$ duty cycle) beam exhibiting a SATA intensity of 0.37 $\mathrm{W} / \mathrm{cm}^{2}$. Although conveniently brief, this application produced minimal skin heating yet facilitated a 3.88-fold increase in steady state caffeine flux. In more general terms, we determined that pulsed ultrasound with a $10 \%$ duty cycle could be just as efficacious for sonophoretic delivery as ultrasound of greater duty cycles. Furthermore, for beams exhibiting a $10 \%$ duty cycle, sonication concurrent with drug deposition was more effective than sonication prior to drug deposition. This last aspect may be of particular importance in the development and testing of novel sonophoretic delivery systems. For further research, it would noteworthy to study if there is an optimal sonophoretic protocol can be generalized into similar hydrophilic and/or lipophilic drug molecules. Also further studies will be necessary to determine the relative contribution of temperature and time parameters in sonophoretic induced- percutaneous enhancement of drug transport.

\section{ACKNOWLEDGEMENTS}

Authors are grateful to Dr Laurence Tetley and Mrs Margaret Mullen of the Integrated Microscopy Facility (Glasgow University, Glasgow, UK) for providing technical assistance.

Also the gratitude is extended to Dr Anthony Gachagan of the Centre of Ultrasonic Engineering (CUE) for providing expertise with ultrasound calibration studies. This research is partly funded by the University of Strathclyde, Glasgow, UK.

\section{REFERENCES}

[1] G. ter Haar, "Therapeutic applications of ultrasound", Prog. Biophys. Mol. Biol., vol. 93, pp. 111-29, 2007.

[2] I.Lavon and J. Kost, "Ultrasound and transdermal drug delivery", Drug Discov. Today, vol. 9, pp. 670-176, 2004.

[3] V. M. Meidan and B. B. Michniak, "Emerging technologies in transdermal therapeutics", Am. J. Ther., vol. 11, pp. 312-16, 2004.

[4] I. Lavon, N. Grossman, J. Kost, E. Kimmel and G. Enden, "Bubble growth within the skin by rectified diffusion might play a significant role in sonophoresis", J. Control. Rel., vol. 117, pp. 246-155, 2007.

[5] E. J. Park, J. Werner and N. B. Smith, "Ultrasound mediated transdermal insulin delivery in pigs using a lightweight transducer", Pharm. Res., vol. 24, pp. 1396-401, 2007.

[6] R. Alvarez-Roman, G. Merino, Y. N. Kalia, A. Naik and R. H. Guy, "Skin permeability enhancement by low frequency sonophoresis; lipid extraction and transport pathways", J. Pharm. Sci., vol. 92, pp. 1138-46, 2003.
I. Lavon, N. Grossman and J. Kost, "The nature of ultrasound-SLS synergism during enhanced transdermal transport", J. Control. Rel., vol. 107, pp. 484-94, 2005.

[8] M. Sivakumar, K. Tachibana, A. B. Pandit, K. Yasui, T. Tuziutiz, A. Towata, Y. Iida, "Transdermal drug delivery using ultrasound theory, understanding and crtitical analysis", Cell Mol. Biol., vol. 51, pp. 767-84, 2005.

[9] S. Mitragotri and J. Kost, "Low frequency sonophoresis: a review", Adv. Drug Deliv. Rev., vol. 56, pp. 589-601, 2004.

[10] G. Merino, Y. N. Kalia and R. H. Guy, "Ultrasound-enhanced transdermal transport", J. Pharm. Sci., vol. 92, pp. 1125-37, 2003

[11] A. Tezel, A. Sens, S. Mitragotri, J. Tuchscherer and S. Mitragotri, "Frequency dependence of sonophoresis", Pharm. Res., vol. 18, pp. 1694-700, 2001.

[12] A. Tezel, A. Sens and S. Mitragotri, "Investigations of the role of cavitation in low frequency sonophoresis using acoustic spectroscopy", J. Pharm. Sci., vol. 91, pp. 444-53, 2002.

[13] S. Paliwal, G. K. Menon and S. Mitragotri, "Low frequency sonophoresis: Ultrastructural basis for stratum corneum permeability assessed using quantum dots", J. Invest. Dermatol., vol. 126, pp. 1095-101, 2006

[14] V. M. Meidan, A. D. Walmsley and W, J. Irwin, "Phonophoresis is it a reality?", Int. J. Pharm., vol. 118, pp. 129-49, 1995.

[15] V. M. Meidan, M. F. Docker, A. D. Walmsley and W. J. Irwin, "Phonophoresis of hydrocortisone with enhancers: an acoustically defined model", Int. J. Pharm., vol. 170, pp. 157-68, 1998.

[16] G. Hayward, G. Benny, R. Banks and W. Galbraith, "The radiation field characteristics of piezoelectric polymer membrane transducers when operating into air", IEEE Transactions UFFC, vol. 47, pp. $1438-47,2000$

[17] G. Benny and G. Hayward, "Beam Profile measurements and simulations for ultrasonic transducers operating in air", J. Acoust. Soc. Am., vol. 107, pp. 2089-100 2000.

[18] A. Gachagan, D. Speirs and A. McNab, "The design of a high power ultrasonic test cell using finite element modelling techniques", Ultrasonics, vol. 41, pp. 283-8, 2003.

[19] J. E. Seto, B. E. Polat, R. V. F. Lopez, D. Blankschtein, R. Langer "Effects of ultrasound and sodium lauryl sulfate on the transdermal delivery of hydrophilic permeants: Comparative in vitro studies with full-thickness and split-thickness pig and human skin," $J$. Cont. Rel., vol. 145, pp. 26-32, 2010.

[20] A. Lesch, C. A. Squier, A. Cruchley, D. M. Williams, and P. "Speight. The permeability of human oral mucosa and skin to water". J. Dent. Res., vol. 68, pp. 1345-9, 1989.

[21] T. J. Franz, Percutaneous absorption: On the relevance of in vitro data. J. Invest. Dermatol., vol. 64, pp. 190-5, 1975.

[22] G. M. Khan, Y. Frum, O. Sarheed, G. M. Eccleston and V. M. Meidan, "Assessment of drug permeability distributions in two different model skins", Int. J. Pharm., vol. 303, pp. 81-7, 2005.

[23] Y. Frum, G. M. Khan, J. Sefcik, J. Rouse, G. M. Eccleston and V. M. Meidan, "Towards a correlation between drug properties and in vitro transdermal flux variability", Int. J. Pharm., vol. 336, pp. 1407, 2007.

[24] R. L. Bronaugh, R. F. Stewart, E. R. Congdon, "Methods for in vitro percutaneous absorption studies II. Animal models for human skin". Toxicol. Appl. Pharmacol., vol. 62, pp. 481-8, 1982.

[25] R. J. Batchelder, R. J. Calder, C. P. Thomas and C. M. Heard, "In vitro transdermal delivery of the major catechins and caffeine from extracts of Camellia sinesis", Int. J. Pharm., vol. 283, pp. 45-51, 2004.

[26] A. Boucaud, L. Machet, B. Arbeille, M.C. Machet, M. Sournac, A. Mavon, F. Patat, and L. Vaillant, "In vitro study of low frequency ultrasound-enhanced transdermal transport of fentanyl and caffeine across human and hairless rat skin", Int. J. Pharm., vol. 228, pp. 69-77, 2001.

[27] A. Boucaud, J. Montharu, L. Machet, B. Arbeille, M.C. Machet, F. Patat, and L. Vaillant, "Clinical, histologic, and electron microscopy study of skin exposed to low-frequency ultrasound", Anat. Rec., vol. 264, pp. 114-9, 2001.

[28] D. Monti, R. Gianelli, P. Chetoni and P. Burglasi, "Comparison of the effect of ultrasound and of chemical enhancers on transdermal permeation of caffeine and morphine through hairless mouse skin in vitro", Int. J. Pharm., vol. 229, pp. 131-7, 2001.

[29] Y. Yuan and R. Verma, "Measuring microelasatic properties of stratum corneum", Coll. Surfaces B Biointerfaces, vol. 48, pp. 6-12, 2006. 
[30] Williams, Ultrasound: biological effects and potential hazards. London: Academic Press, 1983.

[31] R.O. Potts, Physical characterization of the stratum corneum: the relationship of mechanical and barrier properties to lipid and pro- tein structure, In: J. Hadgraft, R.H. Guy, Eds., Transdermal Drug Delivery. Developmental Issues and Research Initiatives, Marcel Dekker, New York pp. 23-57, 1989.

(c) Sarheed and Abdul Rasool; Licensee Bentham Open.

This is an open access article licensed under the terms of the Creative Commons Attribution Non-Commercial License (http://creativecommons.org/licenses/by-nc/3.0/) which permits unrestricted, non-commercial use, distribution and reproduction in any medium, provided the work is properly cited. 